\title{
DERIVATION OF THE LORENTZ BOOST FROM THE EVANS WAVE EQUATION
}

\author{
Myron W. Evans \\ Alpha Institute for Advanced Study (AIAS) \\ E-mail: emyrone@aol.com
}

Received 8 March 2004

The Lorentz boost is derived from the Evans wave equation of generally covariant unified field theory by constructing the Dirac spinor from the tetrad in the $\mathrm{SU}(2)$ representation space of non-Euclidean spacetime. The Dirac equation in its wave formulation is then deduced as a well-defined limit of the Evans wave equation. By factorizing the d'Alembertian operator into Dirac matrices, the Dirac equation in its original first differential form is obtained from the Evans wave equation. Finally, the Lorentz boost is deduced from the Dirac equation using geometrical arguments. A self-consistency check of the Evans wave equation is therefore forged by deducing therefrom the Lorentz boost in the appropriate limit. This procedure demonstrates that the Evans wave equation governs the properties of matter and anti-matter in general relativity and unified field theory and leads both to FermiDirac and Bose-Einstein statistics in general relativity.

Key words: Lorentz boost, Evans wave equation, generally covariant unified field theory.

\section{INTRODUCTION}

General relativity reduces to special relativity when one frame of reference moves at a constant velocity with respect to the other. This well-defined limit is known as the Lorentz boost $[1,2]$. It follows that the recently derived Evans wave equation of generally covariant unified field theory [3-15] must self-consistently and non-trivially reduce to the Lorentz boost, while also suggesting experimentally measurable developments such as the Evans spin field $\mathbf{B}^{(3)}$, observed in the magne- 
tization of matter by circularly or elliptically polarized electromagnetic radiation - the inverse Faraday effect [16]. The Lorentz boost must be derivable analytically from the structure of Evans' generally covariant unified field theory, and therefore the derivation serves as one of many checks available [3-15] on the self-consistency of the Evans theory. The Lorentz boost or transformation was originally devised by Lorentz following a suggestion by Fitzgerald in response to the crisis in physics posed by the Michelson-Morley experiment. It was developed by Lorentz and Poincaré and others in electrodynamics, and then by Einstein in 1905 in his famous theory of special relativity. Ten years later, in 1915, general relativity was proposed by Einstein in the form accepted today, and independently and slightly earlier by Hilbert. The theory of 1915 was as much of a revolutionary advance over its predecessor of 1905 as the latter was over earlier physics. The 1905 special relativity merged space and time, and the 1915 general relativity geometrized natural philosophy using the mathematical methods of Riemann devised in the nineteenth century. Relativity all depends on the Lorentz boost and the slightly earlier Lorentz-Fitzgerald contraction of about 1896.

Einstein was never satisfied that his theory was complete, because the 1915 theory of general relativity is confined to gravitation and spacetime with curvature but no torsion [2] - Riemannian spacetime described with the Christoffel connection. The first generally covariant unified field theory was devised by Evans in 2003 [5-15] using differential geometry to describe spacetime with both curvature and torsion [2]. The Evans theory has been tested experimentally and for analytical self consistency in many ways [5-15] and predicts from spacetime torsion the existence of the fundamental and experimentally well observed Evans spin field of electrodynamics, the $\mathbf{B}^{(3)}$ field. Tiny (nanotesla) $\mathbf{B}^{(3)}$ fields were first observed by Pershan et al. [17], shortly after the laser first became available, as magnetization of matter with circularly polarized electromagnetic radiation at visible frequencies. The existence of $\mathbf{B}^{(3)}$ was first inferred in 1992 by Evans [18] from these and other experimental data such as the magnetization of plasma observed by Deschamps et al [19] with circularly polarized $3.0 \mathrm{GHz}$ radiation. It is now possible to observe a $\mathbf{B}^{(3)}$ field of tens of tesla (i.e., megagauss) magnitude in under dense plasma [20]. It is also known now that in a correctly covariant electrodynamical theory (part of unified field theory) the $\mathbf{B}^{(3)}$ field is responsible for all physical optics, and so is a commonplace observable of everyday experience [3-15]. The revolutionary inference of $\mathbf{B}^{(3)}$ in electrodynamics gradually led to the broader and deeper inference (3-15) of the generally covariant unified field theory sought after by Einstein.

The fundamental concept in the Evans theory is the tetrad [215], which has a well-defined meaning in differential geometry [2], a the- 
ory which shows that the two differential forms that define the gauge invariant fields of nature are the Riemann (curvature) and torsion forms. These are defined, respectively, by the second and first Maurer-Cartan structure relations and in the Evans theory both are synthesized selfconsistently and in an entirely original manner [3-15] from the tetrad form of differential geometry. The tetrad (a vector-valued one-form) is the potential field, the Riemann form (a tensor-valued two-form) is the gauge invariant gravitational field, and the torsion form (a vectorvalued two-form) is the gauge-invariant electromagnetic field, whose fundamental spin invariant is the well observed Evans spin field $\mathbf{B}^{(3)}$. The theory is generally covariant because it is a fully geometrized theory of general relativity, and it is unified because different types of radiation and matter fields all spring from one source, the tetrad.

In Sec. 2 the tetrad is defined in $\mathrm{SU}(2)$ representation space as the $2 \times 2$ transformation matrix between two Pauli spinors. One spinor (a two-component column vector) is defined in the Euclidean orthonormal space [2] of the tetrad, and is labelled $R$ and $L$, and the other in the non-Euclidean base manifold, and is labelled 1 and 2. The tetrad defined in this way has four scalar components (arranged in a $2 \times 2$ matrix) which also define by simple transposition the Dirac spinor (a four-component column vector) in the appropriate special relativistic limit, a limit in which we recover the Dirac wave equation from the Evans wave equation. The labels $R$ and $L$ in the orthonormal space indicate the existence of spin, torsion or handedness and the existence of anti-particles. They are therefore left-handed and right-handed spin labels, and originate in the fact that the tetrad must always be defined as a matrix linking two spaces in such as way as to create spin as well as mass in general relativity. The fundamental reason for this is that there are two Casimir invariants of the Einstein group (and also the Poincaré group): the mass and spin invariants [1,3-15], which in differential geometry are defined respectively by the Riemann and torsion forms, i.e., by the second and first Maurer-Cartan structure relations [2-15], respectively. Only one of these appears in the original 1915 theory, the mass invariant, and then without recognition of the fact that the symmetric metric tensor used by Einstein is the dot product of two, more fundamental, tetrads. The Evans $\mathbf{B}^{(3)}$ field (not inferred until 1992) is now recognized as the fundamental spin invariant of generally covariant electrodynamics, missing entirely from both the Einstein theory of general relativity and the Maxwell-Heaviside theory of special relativity. The fundamental concept of spin, or torsion, is therefore missing entirely from the original 1915 theory of gravitation devised independently by Einstein and Hilbert. Recognition of spin and manifestations thereof such as $\mathbf{B}^{(3)}$ leads to the unified field theory of Evans [3-15]. The geometrical spin properties inherent in the tetrad are enough to lead to the existence of anti-particles in the Evans unified field theory, which is therefore a powerful predictive the- 
ory based on contemporary differential geometry [2-15]. The labels 1 and 2 in the base manifold are the labels of a Pauli spinor in the $\mathrm{SU}(2)$ representation space of the non-Euclidean base manifold. So the Dirac spinor is given meaning in general relativity as a causal property of spacetime, and not as a probability. This interpretation leads straightforwardly to an acceptable Klein-Gordon equation [3-15], the quantized version of the Einstein equation of free particle relativistic momentum. On the other hand the probabilistic interpretation of the Copenhagen School leads in the received opinion [1] to the "abandonment" of the Klein-Gordon equation and a great deal of obscurity and confusion. It is clear now from the Evans unified field theory that it is the obscure ontology of the Copenhagen School that should be abandoned, not the Klein-Gordon equation. This is one of the broader philosophical consequences of the Evans theory in natural philosophy and clearly shows what Einstein and others meant when they rejected the Copenhagen quantum mechanics as incomplete. The Evans unified field theory shows that wave mechanics is a direct result of the tetrad postulate [2], the Evans Lemma and Evans wave and field equations [3-15]. The Evans theory (and copious experimental evidence [13-15] for the theory) shows clearly that wave mechanics is deducible from general relativity and is causal in nature.

In Sec. 3 the original, first differential, form of the Dirac equation is deduced analytically in the special relativistic limit by factorizing the d' Alembertian operator into Dirac matrices [1], making use of the metric tensor in the special relativistic limit.

Finally in Sec. 4 the Lorentz boost is deduced straightforwardly from the Dirac equation using hyperbolic half angles formulae, and the original Lorentz boost matrix recovered geometrically, as required, from the Evans wave equation. These procedures serve as a cross check on the Evans theory, give considerable insight into the meaning of the Dirac spinor in non-Euclidean spacetime and lead to many philosophical ramifications.

\section{DERIVATON OF THE DIRAC SPINOR AND THE DIRAC WAVE EQUATION FROM EVANS' THEORY}

The Evans wave equation is

$$
(\square+k T) q_{\mu}^{a}=0
$$

and is the identity

$$
D^{\mu} D_{\mu} q_{\nu}^{a}=0
$$

based on the tetrad postulate

$$
D_{\mu} q_{\nu}^{a}=0 .
$$


Equation (1) is based on the Evans lemma

$$
\square q_{\mu}^{a}=R q_{\mu}^{a},
$$

which is the purely geometrical result

$$
D^{\mu} D_{\mu}=\square-R,
$$

where $R$ is scalar curvature. In everywhere flat spacetime,

$$
R \rightarrow 0,
$$

where

$$
\square:=\frac{1}{c^{2}} \frac{\partial^{2}}{\partial t^{2}}-\nabla^{2},
$$

is the d'Alembertian operator and where

$$
R=-k T \text {. }
$$

Equation (8) follows from the Evans field equation

$$
(R+k T) q_{\mu}^{a}=0
$$

and applies to all radiated and matter fields, not only gravitation.

In order to derive the Dirac wave equation from Eq. (1), use an $\mathrm{SU}(2)$ representation space and define the tetrad by

$$
\left(\begin{array}{c}
\zeta^{R} \\
\zeta^{L}
\end{array}\right)=\left(\begin{array}{ll}
\phi_{1}^{R} & \phi_{2}^{R} \\
\phi_{1}^{L} & \phi_{2}^{L}
\end{array}\right)\left(\begin{array}{c}
\zeta^{1} \\
\zeta^{2}
\end{array}\right)
$$

Equation (10) may be written more concisely as

$$
\zeta^{a}=q_{\mu}^{a} \zeta^{\mu},
$$

where

$$
\zeta^{a}=\left(\begin{array}{c}
\zeta^{R} \\
\zeta^{L}
\end{array}\right), \quad \zeta^{\mu}=\left(\begin{array}{c}
\zeta^{1} \\
\zeta^{2}
\end{array}\right), \quad q_{\mu}^{a}=\left(\begin{array}{cc}
\phi_{1}^{R} & \phi_{2}^{R} \\
\phi_{1}^{L} & \phi_{2}^{L}
\end{array}\right) .
$$

Here $\zeta^{\mu}$ is a Pauli spinor in the base manifold and $\zeta^{a}$ is a Pauli spinor in the Euclidean orthonormal spacetime used to define the tetrad [2]. The latter is the $2 \times 2$ matrix defined by

$$
q_{\mu}^{a}=\left(\begin{array}{c}
\phi^{R T} \\
\phi^{L T}
\end{array}\right)
$$


where

$$
\begin{aligned}
\phi^{R T} & =\left(\phi_{1}^{R} \phi_{2}^{R}\right), \\
\phi^{L T} & =\left(\phi_{1}^{L} \phi_{2}^{L}\right) .
\end{aligned}
$$

are row vectors transposed from column vectors. We may therefore define the column four-vector

$$
\psi=\left(\begin{array}{c}
\phi^{R} \\
\phi^{L}
\end{array}\right), \quad \phi^{R}=\left(\begin{array}{c}
\phi_{1}^{R} \\
\phi_{2}^{R}
\end{array}\right), \quad \phi^{L}=\left(\begin{array}{c}
\phi_{1}^{L} \\
\phi_{2}^{L}
\end{array}\right),
$$

which obeys the Evans wave equation in the form

$$
(\square+k T) \psi=0 .
$$

Equation (17) is equivalent to the Evans wave equation in the tetrad form

$$
(\square+k T) q_{\mu}^{a}=0
$$

Both Eqs. (17) and (18) lead to the same set of simultaneous equations

$$
\begin{gathered}
\left(\square+k T_{1}^{R}\right) \phi_{1}^{R}=0, \\
\vdots \\
\left(\square+k T_{2}^{L}\right) \phi_{2}^{L}=0 .
\end{gathered}
$$

Now use the principle that general relativity must reduce to special relativity when one frame moves at a constant velocity with respect to the other. In this limit the well-known wave equations of special relativistic quantum mechanics must be recovered from the Evans wave equation. This principle implies [3-15]

$$
k T \rightarrow 1 / \lambda_{C}^{2},
$$

where

$$
\lambda_{C}:=\hbar / m c
$$

is the Compton wavelength of any matter or radiated field.

In this limit, the Evans equation (1) becomes the Dirac wave equation [1]:

$$
\left(\square+m^{2} c^{2} / \hbar^{2}\right) \psi=0 .
$$

The Dirac spinor is therefore recognized as the limiting form of a tetrad whose two rows have been transposed into column two vectors. The Dirac spinor is therefore a geometrical object, not a probability as in 
the Copenhagen interpretation. In the limit (20) there exists a non-zero least or minimum curvature

$$
R_{0}=-(m c / \hbar)^{2}
$$

that defines mass through the Evans principle of least curvature [3-15]. The total curvature

$$
R:=\square-R_{o}=0
$$

vanishes. Each of Eqs. (19) is a Klein-Gordon equation, whose classical limit must be the Einstein equation of relativistic momentum

$$
p^{\mu} p_{\mu}=E^{2} / c^{2}-p^{2}=m^{2} c^{2},
$$

where $E$ is the total relativistic kinetic energy, $p$ the relativistic momentum, and $E_{0}$ the rest energy

$$
E_{0}=m c^{2}
$$

The fact that Eq. (25) must be the classical limit of the Klein-Gordon equation implies the operator equivalence of quantum mechanics

$$
\square=-\left(1 / \hbar^{2}\right) p^{\mu} p_{\mu}
$$

which we have therefore deduced from the Evans unified field theory. Equation (25) is another form [21] of

$$
p=\gamma m v \text {. }
$$

In the non-relativistic limit,

$$
p=m v, \quad T=\frac{1}{2} m v^{2},
$$

which are the Newton equations for momentum and kinetic energy of a free particle.

The famous equations of dynamics are therefore a consequence of the Evans principle of least curvature [3-15]. The total curvature vanishes in the Klein-Gordon equation and Dirac equation, but the individual components $\square$ and $R_{0}$ do not vanish. This result means that mass is a form of curvature and is defined for any elementary particle (including the neutrino and photon) by the least curvature (23). The Dirac spinor is defined in terms of the tetrad, and so the Dirac spinor introduces spin into the definition of a particle, as discussed in Sec. 1. Spin is introduced geometrically through the definition (10), a definition which implies that the famous half integral spin is spacetime torsion in $\mathrm{SU}(2)$ representation space. Eq. (10) means that each 
elementary particle is converted by the parity operator into its antiparticle, with the same mass but opposite handedness or helicity. The helicities originate in the Pauli spinor

$$
\zeta^{a}=\left(\begin{array}{l}
\zeta^{R} \\
\zeta^{L}
\end{array}\right)
$$

where

$$
a=L, R
$$

are labels of the orthonormal space needed to define the tetrad. Analogously, in $\mathrm{O}(3)$ electrodynamics, these spin labels become

$$
a=(1),(2),(3)
$$

of the complex circular basis [3-15], indicating three states of spin for the photon (the transverse (1) and (2) and the longitudinal (3)), three sets of field equations in the appropriate limit, and giving the $\mathbf{B}^{(3)}$ field from general relativity.

These spin states are missing from Einstein's generally covariant theory of gravitation but are present in the Evans wave and field equations of generally covariant unified field theory.

The spin states are also missing from Einstein's theory of special relativity and Newtonian dynamics. They are observed experimentally however in numerous ways, for example the anomalous Zeeman effect, atomic and molecular spectra. ESR, NMR, MRI, the existence of antiparticles, in Fermi-Dirac statistics, and so on. The Evans theory shows that these properties of nature are due to spacetime geometry in a particular limit, Eq. (20). The spin states are interconverted by the parity operator:

$$
\left(\begin{array}{l}
\zeta^{R} \\
\zeta^{L}
\end{array}\right) \rightarrow\left(\begin{array}{l}
\zeta^{L} \\
\zeta^{R}
\end{array}\right) .
$$

Equation (23) gives the important additional insight that mass vanishes in an everywhere flat spacetime. If mass vanishes, then so does rest energy and relativistic momentum, showing that the total relativistic kinetic energy $E$ also vanishes in an everywhere flat spacetime. Therefore it makes no sense to assert the existence of a particle without mass, because such as particle would not have any kinetic energy and would not exist experimentally. Therefore there can be no massless neutrino and no massless photon. This is now known to be a consequence of the least curvature principle of Evans [3-15] and also a consequence of the Evans wave equation. In the appropriate limit the latter reduces to the correctly covariant form of the Proca equation [1,3-15] for the photon with mass. Einstein's special relativistic theory is not therefore a 
theory in which spacetime is everywhere flat, because in such a spacetime there can be no mass, energy, spin, charge density and current density anywhere in the universe. For every physical theory therefore, the operator $D^{\mu} D_{\mu}$ never becomes $\square$ identically. This statement is an expression of the Evans lemma.

\section{DERIVATION OF THE DIRAC EQUATION AS A FIRST-ORDER DIFFERENTIAL EQUATION}

Dirac originally inferred his famous equation as a first order differential equation. In this section we deduce this from the Dirac wave equation (22), which is a limit of the Evans wave equation as demonstrated already. The starting point is

$$
\partial^{\mu}=g^{\mu \nu} \partial_{\nu}
$$

where $g^{\mu \nu}$ is the Minkowskian metric tensor in the special relativistic limit [1]. Using Eq. (34), the d'Alembertian operator is

$$
\square=\partial^{\mu} \partial_{\mu}=g^{\mu \nu} \partial_{\nu} \partial_{\mu}
$$

The metric is now factorized into the anticommutator of $4 \times 4 \gamma^{\mu}$ matrices [1]

$$
g^{\mu \nu}=\gamma^{\mu} \gamma^{\nu}=\frac{1}{2}\left\{\gamma^{\mu}, \gamma^{\nu}\right\}
$$

so Eq. (22) becomes

$$
\left(\gamma^{\mu} \gamma^{\nu} \partial_{\nu} \partial_{\mu}+(m c / \hbar)^{2}\right) \psi=0
$$

Now factorize the operator

$$
\begin{aligned}
& \left(i \gamma^{\nu} \partial_{\nu}+m c / \hbar\right)\left(i \gamma^{\mu} \partial_{\mu}-m c / \hbar\right) \\
& =-\gamma^{\mu} \gamma^{\nu} \partial_{\mu} \partial_{\nu}-(m c / \hbar)^{2} .
\end{aligned}
$$

It follows that

$$
\left(i \gamma^{\nu} \partial_{\nu}+m c / \hbar\right)\left(i \gamma^{\mu} \partial_{\mu}-m c / \hbar\right) \psi=0
$$

and that

$$
\left(i \gamma^{\mu} \partial_{\mu}-m c / \hbar\right) \psi=0
$$

Finally, use the operator equivalence

$$
p_{\mu}=i \hbar \partial_{\mu}
$$


to obtain

$$
\left(\gamma^{\mu} p_{\mu}-m c\right) \psi=0 .
$$

Equation (40) is the Dirac equation in differential form, i.e., in representation space [1], and Eq. (42) is the Dirac equation in momentum space.

The $\gamma^{\mu}$ matrices are identified as the $4 \times 4$ Dirac matrices

$$
\gamma^{0}=\left(\begin{array}{ll}
0 & 1 \\
1 & 0
\end{array}\right), \quad \gamma^{i}=\left(\begin{array}{cc}
0 & -\sigma^{i} \\
\sigma^{i} & 0
\end{array}\right)
$$

where

$$
\sigma^{1}=\left(\begin{array}{ll}
0 & 1 \\
1 & 0
\end{array}\right), \quad \sigma^{2}=\left(\begin{array}{cc}
0 & -i \\
i & 0
\end{array}\right), \quad \sigma^{3}=\left(\begin{array}{cc}
1 & 0 \\
0 & -1
\end{array}\right)
$$

are the Pauli matrices.

Recall that

$$
\psi=\left(\begin{array}{c}
\phi^{R} \\
\phi^{L}
\end{array}\right)=\left(\begin{array}{c}
\phi_{1}^{R} \\
\phi_{2}^{R} \\
\phi_{1}^{L} \\
\phi_{2}^{L}
\end{array}\right) .
$$

Using Eq. (43)-(45) in Eq. (42) gives

$$
\begin{aligned}
& m c^{2} \phi^{R}=(E+\boldsymbol{\sigma} \cdot \mathbf{p} c) \phi^{L}, \\
& m c^{2} \phi^{L}=(E-\boldsymbol{\sigma} \cdot \mathbf{p} c) \phi^{R},
\end{aligned}
$$

where Eq. (47) is the parity inverted Eq. (46). The Dirac equation is therefore a relation between the Pauli spinors $\phi^{R}$ and $\phi^{L}$, which in turn are derived from the tetrad, the eigenfunction of the Evans wave equation and Evans lemma.

\section{DERIVATION OF THE LORENTZ BOOST}

The Dirac equations (46) and (47) may be written in the simple form

$$
\begin{gathered}
\phi^{R}=e^{\boldsymbol{\sigma} \cdot \boldsymbol{\theta}} \phi^{L}, \\
\phi^{L}=e^{-\boldsymbol{\sigma} \cdot \boldsymbol{\theta}} \phi^{R},
\end{gathered}
$$

where

$$
e^{\boldsymbol{\sigma} \cdot \boldsymbol{\theta}}=\cos h \theta+\boldsymbol{\sigma} \cdot \mathbf{n} \sin h \theta,
$$




$$
e^{-\boldsymbol{\sigma} \cdot \boldsymbol{\theta}}=\cos h \theta-\boldsymbol{\sigma} \cdot \mathbf{n} \sin h \theta .
$$

Here

$$
\begin{gathered}
\cos h \theta=E / m c^{2}=\gamma, \\
\sin h \theta=p c / m c^{2}=\beta \gamma .
\end{gathered}
$$

The Einstein equation (25) follows with these definitions and the hyperbolic angle formula

$$
\cos h^{2} \theta-\sin h^{2} \theta=1 .
$$

The parameters $\beta$ and $\gamma$ are defined by

$$
\begin{gathered}
\gamma^{2}\left(1-\beta^{2}\right)=1, \\
\beta=v / c, \quad \gamma=\left(1-v^{2} / c^{2}\right)^{-1 / 2},
\end{gathered}
$$

where $v$ is the constant velocity of one frame with respect to another in special relativity.

Now write the Dirac equations are

$$
\begin{gathered}
\phi^{R}=e^{(\boldsymbol{\sigma} \cdot \boldsymbol{\theta}) / 2}\left(e^{(\boldsymbol{\sigma} \cdot \boldsymbol{\theta}) / 2} \phi^{L}\right), \\
\phi^{L}=e^{-(\boldsymbol{\sigma} \cdot \boldsymbol{\theta}) / 2}\left(e^{-(\boldsymbol{\sigma} \cdot \boldsymbol{\theta}) / 2} \phi^{R}\right),
\end{gathered}
$$

and define

$$
\begin{gathered}
\phi^{R}(p)=e^{(\boldsymbol{\sigma} \cdot \boldsymbol{\theta}) / 2} \phi^{R}(0), \\
\phi^{L}(p)=e^{-(\boldsymbol{\sigma} \cdot \boldsymbol{\theta}) / 2} \phi^{L}(0),
\end{gathered}
$$

to obtain the Lorentz boost [1]

$$
\psi(p)=\left(\begin{array}{cc}
e^{\boldsymbol{\sigma} \cdot \boldsymbol{\theta} / 2} & 0 \\
0 & e^{-\boldsymbol{\sigma} \cdot \boldsymbol{\theta} / 2}
\end{array}\right) \psi(0) .
$$

The Lorentz boost is therefore a transformation between the Dirac spinors $\psi(p)$ and $\psi(0)$, and the Dirac equation is

$$
\psi(p)=\left(\begin{array}{cc}
e^{\boldsymbol{\sigma} \cdot \boldsymbol{\theta}} & 0 \\
0 & e^{-\boldsymbol{\sigma} \cdot \boldsymbol{\theta}}
\end{array}\right) \psi^{* *}(p),
$$

where

$$
\psi^{* *}=\hat{P}(\psi)
$$

is the parity inverted Dirac spinor.

From Eqs. (61) and (62), it is seen that there is a simple geometrical relation between the Dirac equation and the Lorentz boost [1], 
one may be constructed from the other using hyperbolic half angle formulae. It follows that the Lorentz boost is a well-defined geometrical limit of the Evans wave equation, which is what we set out to prove.

Finally recognize that [1]

$$
\mathbf{K}= \pm i \boldsymbol{\sigma} / 2
$$

where

$$
K_{z}=\left.\frac{1}{i} \frac{\partial B}{\partial \theta}\right|_{\theta=0}
$$

and where

$$
B=\left(\begin{array}{cccc}
\cosh \theta & \sinh \theta & 0 & 0 \\
\sinh \theta & \cosh \theta & 0 & 0 \\
0 & 0 & 1 & 0 \\
0 & 0 & 0 & 1
\end{array}\right)
$$

is the famous Lorentz boost matrix. By recognizing that the parameter $\theta$ of the Lorentz boost also appears in the Dirac equation, it can be seen that the Lorentz boost is derivable from the Evans wave equation. The unified field theory reduces to the most important and earliest inference of all relativity theory, the Lorentz-Fitzgerald contraction.

Acknowledgments. The Ted Annis Foundation, Applied Science Associates, Craddock Inc., and ADAS are thanked for generous funding. The staff of AIAS is thanked for many interesting discussions.

\section{REFERENCES}

1. L. H. Ryder, Quantum Field Theory 2nd edn. (Cambridge University Press, 1996).

2. S. M. Caroll, Lecture Notes in General Relativity, a graduate course in Univ. California, Santa Barbara, arXiv:gr-gq/9712019 vl 3 Dec 1997, available from the author.

3. M. W. Evans, "A unified field equation for gravitation and electromagnetism," Found. Phys. Lett. 16, 367 (2003).

4. M. W. Evans, "A generally covariant wave equation for grand unified field theory," Found. Phys. Lett. 16, 507 (2003).

5. M. W. Evans, "The equations of grand unified field theory in terms of the Maurer-Cartan structure relations of differential geometry," Found. Phys. Lett. 17, 25 (2004).

6. M. W. Evans, "Derivation of Dirac's equation from the Evans wave equation," Found. Phys. Lett. 17, 149 (2004). 
7. M. W. Evans, "Unification of the gravitational and strong nuclear fields," Found. Phys. Lett. 17, 267 (2004).

8. M. W. Evans, "The Evans lemma of differential geometry," Found. Phys. Lett. 17, 433 (2004), in press; preprint on www.aias.us.

9. M. W. Evans, "Derivation of the wave equation from the Lagrangian and action. Origin of the Planck constant in general relativity," Found. Phys. Lett. 17, 535 (2004), in press; preprint on www.aias.us.

10. M. W. Evans and AIAS Author Group, "Development of the Evans equation in the weak-field limit, the electrogravitic equation," Found. Phys. Lett. 17, 497 (2004), in press.

11. M. W. Evans, "Physical optics, the Sagnac effect, and the Aharonov Bohm effect in the Evans unified field theory," Found. Phys. Lett. 17, 301 (2004), preprint on www.aias.us.

12. M. W. Evans, "Derivation of the geometrical phase from the Evans phase law of generally covariant unified field theory," Found. Phys. Lett. 17, 393 (2004); preprint on www.aias.us.

13. M W. Evans, "New concepts from the Evans unified field theory. Part One: The evolution of curvature, oscillatory universe without singularity, causal quantum mechanics and general force and field equations," Found. Phys. Lett., submitted; preprint on www.aias.us.

14. M. W. Evans, "New concepts from the Evans unified field theory." Part Two: Derivation of the Heisenberg equation and replacement of the Heisenberg uncertainty principle," Found. Phys. Lett., submitted; preprint on www.aias.us.

15. L. Felker, ed., The Evans Equations of Unified Field Theory (World Scientific, Singapore, in preparation, 2004/2005). M. W. Evans, ed., The Collected Scientific Papers of Myron Wyn Evans (Artspeed website, from 2004), Vols. 1 and 2, the years 2000 to 2004; Modern Nonlinear Optics, a special topics issue in three parts of Advances in Chemical Physics, I. Prigogine and AS. A. Rice, series eds. (Wiley Interscience, New York, 2001, 2nd and e-book editions), Vols. 119(1)-119(3); ibid., 1st edn., Vols. 85(1)85(3) (1992, 1993, and 1997, hardback and softback editions). M. W. Evans and L. B. Crowell, Classical and Quantum Electrodynamics and the $\mathbf{B}^{(3)}$ Field (World Scientific, Singapore, 2001). M. W. Evans et al., The Enigmatic Photon (Kluwer Acadmic, Dordrecht, 1994 to 2004, hardback and softback editions), five volumes. M. W. Evans and A. A. Hasanein, The Photomagneton in Quantum Field Theory (World Scientific, Singapore, 1994).

16. J. P. van der Ziel, P. Pershan, and L. D. Malmstrom, Phys. Rev. Lett. 15, 190 (1965).

17. P. S. Pershan, J. P. van der Ziel, and L. D. Malmstrom, Phys. Rev. 143, 574 (1966). 
18. M. W. Evans, Physica B 182, 227, 237 (1992).

19. J. Deschamps, M. Fitaire, and M. Lagoutte, Phys. Rev. Lett. 25, $1330(1970)$.

20. M. Tatarikis et al., "Measurement of the inverse Faraday effect in high-intensity laser produced plasmas" (CFL Annual Report Rutherford Appleton Laboratory, 1998 1999, m.tatarikis@ic.ac.uk).

21. J. B. Marion and S. T. Thornton, Classical Dynamics of Particles and Systems, 3rd edn. (HBJ, New York, 1998). 